\title{
One-pot aromatic bromination-rearrangement catalyzed by $\mathrm{GaCl}_{3}$
}

\author{
Mieko Arisawa, Atsushi Suwa, Masanori Ashikawa, and Masahiko Yamaguchi* \\ Department of Organic Chemistry, Graduate School of Pharmaceutical Sciences, \\ Tohoku University, Aoba, Sendai 980-8578, Japan \\ E-mail: yama@mail.pharm.tohoku.ac.jp
}

\section{This paper is dedicated to Prof. Keiichiro Fukumoto in recognition of his outstanding contributions to organic chemistry}

(received 08 Mar 03; accepted 26 May 03; published on the web 29 May 03)

\begin{abstract}
Reaction of monoalkylbenzenes with bromine in the presence of a catalytic amount of $\mathrm{GaCl}_{3}$ (5 mol \%) initially gives $o / p$-bromination products, which are converted into mixtures containing considerable amounts of the $m$-brominated products. Notably, the bromination of dimethyl-, trimethyl-, and tetramethylbenzenes gives dibromo- and/or tribromoarenes, which are converted into monobromoarenes.
\end{abstract}

Keywords: Gallium trichloride, aromatic bromination, isomerization

\section{Introduction}

The bromination of aromatic compounds has been conducted using halogenating reagents such as $\mathrm{Br}_{2}$ and $\mathrm{HOBr}$ in the presence or absence of catalysts. ${ }^{1}$ These methods convert monoalkylbenzenes into $o / p$-brominated products. Since the $m$-derivatives are formed in small amounts, their preparations in general employ multistep processes. ${ }^{2}$ A previous report that $\mathrm{Al}_{2} \mathrm{Cl}_{6}$-water catalyst promotes the isomerization of bromoarenes ${ }^{3}$ led us to study one-pot catalytic procedures for the $o / p$ - bromination of monoalkylbenzenes followed by isomerization to give mixtures containing the $m$ - derivatives. ${ }^{4}$ We now show that $\mathrm{GaCl}_{3}{ }^{5,6,7}$ catalyzes such bromination of alkylbenzenes. Also, it was found that the bromination of dimethyl-, trimethyl-, and tetramethylbenzenes initially gives dibromo- and/or tribromoarenes, which are converted into monobromoarenes.

\section{Results and Discussion}


To a solution of hexylbenzene and $\mathrm{GaCl}_{3}$ (5 mol \%) in methylcyclohexane was added an equimolar amount of bromine, and the mixture was stirred at room temperature for $12 \mathrm{~h}$ giving the bromohexylbenzenes $o \mathbf{- 1}, \mathbf{m - 1}$, and $p-\mathbf{1}$ in $18 \%, 38 \%$, and $15 \%$ yields, respectively (Table 1 , entry 3). The structures were determined by comparison with the authentic samples prepared separately (see Experimental Section). Benzyl bromination proceeded in the absence of the catalyst, and 1 was not detected. The use of $\mathrm{FeCl}_{3}$ (3.6 mol \%) exhibited normal orientations, giving $o-1$ (20\%) and p-1 (78\%). Such aromatic bromination isomerization could also be carried out with $\mathrm{AlCl}_{3}$ (5 mol \%), although the reaction was sometimes not reproducible, probably because of the insolubility of $\mathrm{AlCl}_{3}$ in this solvent. The bromination using $\mathrm{GaCl}_{3}$ is rapid, and $o$ $\mathbf{1}$ and $p-\mathbf{1}$ are obtained in $5 \mathrm{~min}$ in $23 \%$ and $42 \%$ yields, respectively, with a very small amount of $m \mathbf{- 1}$ (entry 4). When the mixture is stirred for $12 \mathrm{~h}$ at room temperature, $p-\mathbf{1}$ decreases and $m-\mathbf{1}$ increases. Bromination of several alkylbenzenes catalyzed by $\mathrm{GaCl}_{3}$ is shown in Table 1 . In the case of neopentylbenzene and isobutylbenzene, larger amounts of the $m$-derivatives are obtained, which may be a result of steric reasons (entries 6 and 7). The different $o / p$ ratio of toluene and ethylbenzene may also be explained analogously (entries 1 and 2): a methyl group behaves as a considerably smaller group than an ethyl group in this reaction.

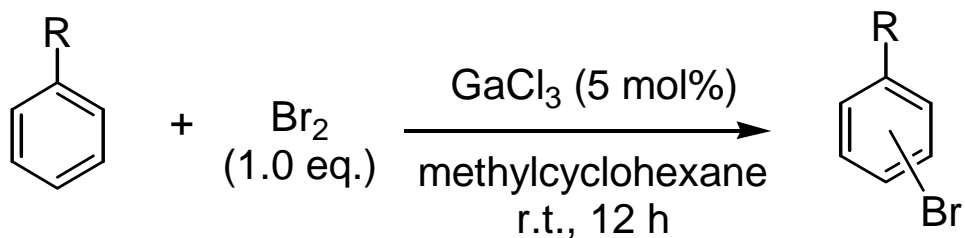

Table 1. Bromination of alkylbenzenes catalyzed by $\mathrm{GaCl}_{3}$

\begin{tabular}{|c|c|c|c|c|}
\hline \multirow{2}{*}{ Entry } & \multirow{2}{*}{$\mathrm{R}$} & \multicolumn{3}{|c|}{ Yield $^{\mathrm{a}}(\%)$} \\
\hline & & $O-$ & $m-$ & $p-$ \\
\hline 1 & $\mathrm{CH}_{3}$ & 32 & 32 & 11 \\
\hline 2 & $\mathrm{CH}_{3} \mathrm{CH}_{2}$ & 16 & 32 & 14 \\
\hline 3 & $\mathrm{CH}_{3}\left(\mathrm{CH}_{2}\right)_{4} \mathrm{CH}_{2}$ & 18 & 38 & 15 \\
\hline $4^{\mathrm{a}}$ & $\mathrm{CH}_{3}\left(\mathrm{CH}_{2}\right)_{4} \mathrm{CH}_{2}$ & 23 & 3 & 42 \\
\hline 5 & $\mathrm{CH}_{3}\left(\mathrm{CH}_{2}\right)_{6} \mathrm{CH}_{2}$ & 23 & 34 & 20 \\
\hline 6 & $\left(\mathrm{CH}_{3}\right)_{2} \mathrm{CHCH}_{2}$ & 21 & 49 & 19 \\
\hline 7 & $\left(\mathrm{CH}_{3}\right)_{3} \mathrm{CCH}_{2}$ & 8 & 62 & 22 \\
\hline
\end{tabular}

${ }^{\mathrm{a}}$ Determined by ${ }^{1} \mathrm{H}-\mathrm{NMR}$. ${ }^{\mathrm{b}}$ The reaction was conducted for $5 \mathrm{~min}$.

Polymethylbenzenes exhibit interesting behaviors: polybrominated products are initially formed, which are converted into monobromides. Reaction of $m$-xylene for $1 \mathrm{~min}$ gives the 4,6dibrominated 4,6-3 as the major product, and the monobromide 2 predominates after $12 \mathrm{~h}$ (Table 
2). Such a phenomenon has not been reported before. Bromination of $m$-xylene with $\mathrm{AlCl}_{3}$ under the same conditions for 1 min gives 4-2 predominantly, which is the usual orientation.

Similar tendencies are observed in the bromination of $p$-xylene with $\mathrm{GaCl}_{3}$ (Table 3 ). The reaction for 5 min gives 2,5-dibrominated 5 predominantly, which is converted into 2brominated 4 after $12 \mathrm{~h}$. In this case, $\mathrm{AlCl}_{3}$ also shows somewhat related isomerization behaviors, although less prominently than with $\mathrm{GaCl}_{3}$.

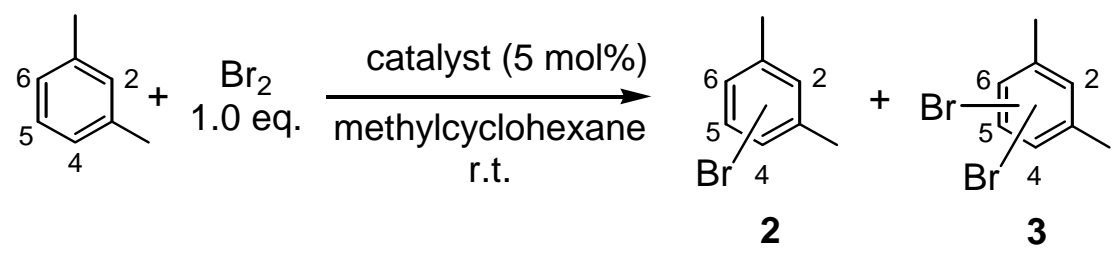

Table 2. Bromination of $m$-Xylene

$$
\text { Yield }^{\mathrm{a}}(\%)
$$

\begin{tabular}{|c|c|c|c|c|c|c|c|c|}
\hline \multirow[t]{2}{*}{ Catalyst } & \multirow[t]{2}{*}{ Time } & \multicolumn{4}{|c|}{2} & \multicolumn{3}{|c|}{3} \\
\hline & & Position $^{\mathrm{b}}$ & $2-$ & 4- & 5- & $2,4-$ & $2,5-$ & $4,6-$ \\
\hline \multirow{2}{*}{$\mathrm{GaCl}_{3}$} & $1 \mathrm{~min}$ & & 5 & 15 & trace & 24 & trace & 40 \\
\hline & $12 \mathrm{~h}$ & & 11 & 30 & 19 & 2 & 11 & 18 \\
\hline $\mathrm{AlCl}_{3}$ & $1 \mathrm{~min}$ & & 9 & 83 & trace & $\mathrm{nd}^{\mathrm{c}}$ & $\mathrm{nd}^{\mathrm{c}}$ & trace \\
\hline
\end{tabular}

${ }^{\mathrm{a}}$ Based on bromine, as determined by ${ }^{1} \mathrm{H}-\mathrm{NMR}$. ${ }^{\mathrm{b}}$ Bromide positions. Numbering based on the starting $m$-xylene. ${ }^{\mathrm{C}}$ Not detected by GC-MS.

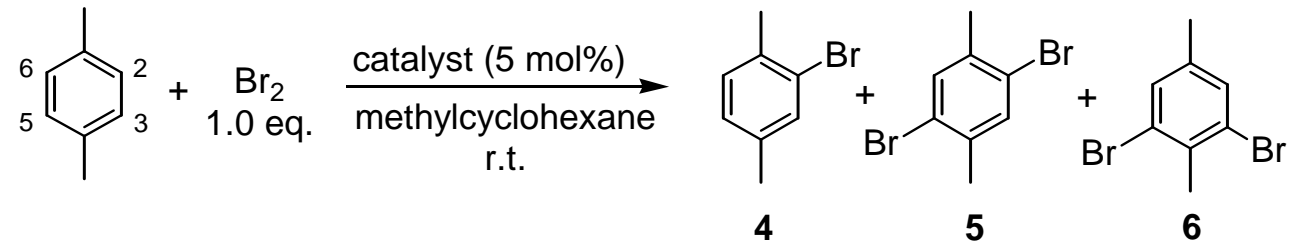

Table 3. Bromination of $p$-Xylene

\begin{tabular}{ccccc}
\hline \multirow{2}{*}{ Catalyst } & Time & $\mathbf{4}$ & Yield $^{\mathrm{a}}(\%)$ \\
\hline \multirow{2}{*}{$\mathrm{GaCl}_{3}$} & $5 \mathrm{~min}$ & 20 & 64 & $\mathbf{6}$ \\
& $12 \mathrm{~h}$ & 64 & 28 & 6 \\
$\mathrm{AlCl}_{3}$ & $5 \mathrm{~min}$ & 47 & 46 & 6 \\
& $12 \mathrm{~h}$ & 67 & 29 & 3 \\
\hline
\end{tabular}

${ }^{\mathrm{a}}$ Based on bromine, as determined by ${ }^{1} \mathrm{H}-\mathrm{NMR}$. 
Bromination of 1,2,3- and 1,3,5-trimethylbenzene with $\mathrm{GaCl}_{3}$ initially gives considerable amounts of dibromo- and tribromoarenes, which are converted into monobromoarenes (Table 4). It should be noted that 5-bromo-1,2,3-trimethylbenzene can be prepared in one step from the corresponding hydrocarbon. The previous preparation of this compound employed a multistep process. ${ }^{8}$ Reactions of both 1,2,3,4-tetramethyl- and 1,2,4,5-tetramethylbenzene with $\mathrm{GaCl}_{3}$ give initially equal amounts of monobromides and dibromides, which are converted into the monobromides after $12 \mathrm{~h}$ (Table 4). Thus, the second and/or the third brominations are faster than the first bromination in the reactions catalyzed by $\mathrm{GaCl}_{3}$. It seems that the bromide group behaves as an activating group in the electrophilic substitution. We propose that the interaction of $\mathrm{GaCl}_{3}$ with $\mathrm{Br}$, as well as the $\mathrm{C}-\mathrm{H}$ bonds of neighboring methyl groups, promote such polybromination. Previously, we reported aromatic substitution reactions which involve interactions between $\mathrm{GaCl}_{3}$ and $\mathrm{C}-\mathrm{H}$ bonds. ${ }^{5}$ The monobrominated arenes obtained here are the thermodynamically controlled products, because of the lower numbers of $o$ - interactions between the bromide and the methyl group.

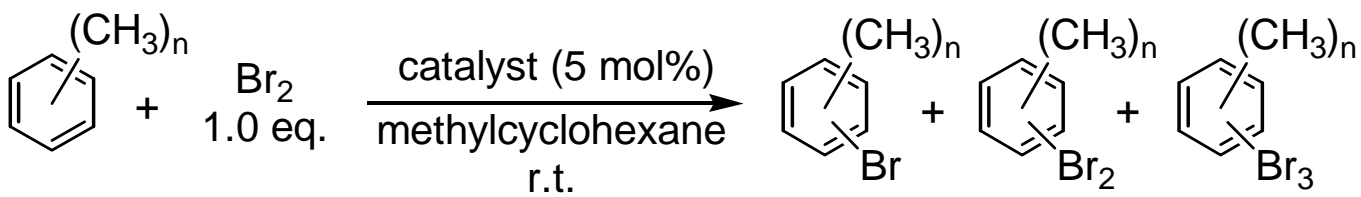

Table 4. Bromination of tri- and tetramethylbenzenes

\begin{tabular}{|c|c|c|c|c|c|}
\hline \multirow{2}{*}{ Substrate } & \multirow{2}{*}{ Catalyst } & \multirow{2}{*}{ Time } & \multicolumn{3}{|c|}{ Yield $^{\mathrm{a}}(\%)$} \\
\hline & & & Monobromide & Dibromide & Tribromide \\
\hline \multirow{2}{*}{ 1,2,3-Trimethylbenzene } & \multirow{2}{*}{$\mathrm{GaCl}_{3}$} & $1 \mathrm{~min}$ & $18(4), 15(5)$ & $25(4,6)$ & 42 \\
\hline & & $12 \mathrm{~h}$ & 22 (4), 50 (5) & $10(4,6)$ & $\mathrm{nd}^{\mathrm{b}}$ \\
\hline \multirow{3}{*}{ 1,3,5-Trimethylbenzene } & \multirow{2}{*}{$\mathrm{GaCl}_{3}$} & $1 \mathrm{~min}$ & 16 & 54 & 27 \\
\hline & & $12 \mathrm{~h}$ & 40 & 34 & 22 \\
\hline & $\mathrm{AlCl}_{3}$ & $1 \mathrm{~min}$ & 84 & 14 & Trace \\
\hline \multirow{2}{*}{ 1,2,3,4-Tetramethylbenzene } & \multirow{2}{*}{$\mathrm{GaCl}_{3}$} & $1 \mathrm{~min}$ & 52 & 41 & - \\
\hline & & $12 \mathrm{~h}$ & 90 & 4 & - \\
\hline \multirow{3}{*}{ 1,2,4,5-Tetramethylbenzene } & \multirow{2}{*}{$\mathrm{GaCl}_{3}$} & $1 \mathrm{~min}$ & 50 & 41 & - \\
\hline & & $12 \mathrm{~h}$ & 71 & 26 & - \\
\hline & $\mathrm{AlCl}_{3}$ & $1 \mathrm{~min}$ & 76 & 22 & - \\
\hline
\end{tabular}

${ }^{\mathrm{a}}$ Based on bromine, as determined by ${ }^{1} \mathrm{H}$-NMR. In parentheses is the brominated position. Numbering based on starting trialkylbenzene. ${ }^{b}$ Not detected by GC-MS.

\section{Experimental Section}


Typical procedures for bromination. Under an argon atmosphere, a $1.0 \mathrm{M}$ solution of $\mathrm{GaCl}_{3}$ in methylcyclohexane $(0.25 \mathrm{~mL}, 5 \mathrm{~mol} \%)$ was added to a methylcyclohexane $(7.5 \mathrm{~mL})$ solution of hexylbenzene $(0.90 \mathrm{~mL}, 5.0 \mathrm{mmol})$ at room temperature. After $5 \mathrm{~min}$, bromine $(0.25 \mathrm{~mL}$, $5.0 \mathrm{mmol}$ ) was added, and the mixture was stirred for $12 \mathrm{~h}$. Then saturated aqueous $\mathrm{Na}_{2} \mathrm{SO}_{3}$ was added, and the organic materials were extracted three times with hexane, the extracts dried over $\mathrm{MgSO}_{4}$, and concentrated. The residue was purified by silica gel chromatography ( $n$-hexane) to give a mixture (852 mg) of $o-\mathbf{1}, \mathbf{m - 1}$, and $p-\mathbf{1}$ in $18 \%, 38 \%$, and $15 \%$ yield, respectively. ${ }^{1} \mathrm{H}-$ NMR (400 MHz, $\mathrm{CDCl}_{3}$ ): $\delta$ 0.88-0.91 (9H, m, $o, m, p$ ), 1.29-1.39 (18H, m, o, $m, p$ ), 1.55-1.62 (6H, m, o, $m, p), 2.52-2.58(4 \mathrm{H}, \mathrm{m}, m, p), 2.71(2 \mathrm{H}, \mathrm{t}, J=8.0 \mathrm{~Hz}, o), 7.00-7.05(\mathrm{~m}, 1 \mathrm{H}, o, 2 \mathrm{H}$, p), $7.06(1 \mathrm{H}, \mathrm{d}, J=8.8 \mathrm{~Hz}, m), 7.13(1 \mathrm{H}, \mathrm{t}, J=8.0 \mathrm{~Hz}, m), 7.19-7.21(2 \mathrm{H}, \mathrm{m}, o), 7.29(1 \mathrm{H}, \mathrm{d}, J=$ $7.2 \mathrm{~Hz}, m), 7.34(1 \mathrm{H}, \mathrm{s}, m), 7.37(2 \mathrm{H}, \mathrm{d}, J=8.8 \mathrm{~Hz}, p), 7.51(1 \mathrm{H}, \mathrm{d}, J=8.0 \mathrm{~Hz}, o) .{ }^{13} \mathrm{C}-\mathrm{NMR}$ (100 MHz, $\left.\mathrm{CDCl}_{3}\right): \delta 14.2(o, m, p), 22.7(m, p), 22.7(o), 29.0(p), 29.0(m), 29.2(o), 30.0(o)$, $31.3(\mathrm{~m}), 31.4(p), 31.8(o, m, p), 35.4(p), 35.7(m), 36.3(o), 119.1(p), 122.2(m), 124.3(o)$, $126.9(\mathrm{~m}), 127.1(\mathrm{o}), 127.2(\mathrm{o}), 128.5(\mathrm{~m}), 129.6(\mathrm{~m}), 130.0(\mathrm{o}), 130.1(\mathrm{o}), 131.1(\mathrm{p}), 131.3(\mathrm{~m})$, $132.6(o), 141.7(p), 142.0(o), 145.1(m)$. IR (neat) 2955, 2927, 2856, 1595, 1568, 1488, 1469, 1378, 1072, 1024, 1011, 774, 749, $692 \mathrm{~cm}^{-1}$. MS (EI) $\mathrm{m} / \mathrm{z} 242\left(\mathrm{M}^{+}+2,37\right), 240\left(\mathrm{M}^{+}, 38\right), 172$ ( $\left.\mathrm{M}^{+}-68,54\right), 171\left(\mathrm{M}^{+}-69,71\right), 169\left(\mathrm{M}^{+}-71,68\right), 91\left(\mathrm{M}^{+}-149,100\right), 43\left(\mathrm{M}^{+}-197,63\right)$. HRMS. Calcd for $\mathrm{C}_{12} \mathrm{H}_{17} \mathrm{Br}$ : 240.0513. Found: 240.0523. Anal. Calcd for $\mathrm{C}_{12} \mathrm{H}_{17} \mathrm{Br}$ : C; 59.76, H; 7.10, Br; 33.13\%. Found: C; 59.60, H; 7.09, Br; 33.28\%.

Preparation of 1-bromo-3-hexylbenzene. Under an argon atmosphere, a 1.6 $M$ hexane solution of $n$-BuLi (44 mL, $70 \mathrm{mmol})$ was added to a THF solution $(100 \mathrm{~mL})$ of 1 pentyltriphenylphosphonium bromide $(24.8 \mathrm{~g}, 60 \mathrm{mmol})$ at $-78^{\circ} \mathrm{C}$. After stirring for $1 \mathrm{~h}$ at room temperature, $m$-bromobenzaldehyde $(5.83 \mathrm{~mL}, 50 \mathrm{mmol})$ was added at $-78{ }^{\circ} \mathrm{C}$, and the mixture was stirred for $2 \mathrm{~h}$ at room temperature, then saturated aq. $\mathrm{NH}_{4} \mathrm{Cl}$ was added. The organic materials were extracted three times with $n$-hexane, dried over $\mathrm{MgSO}_{4}$ and concentrated. Flash chromatography over silica gel ( $n$-hexane) gave 1-bromo-3-(2-hexenyl)benzene $(9.24 \mathrm{~g}, 78 \%$, $E: Z=2: 5)$. Under a hydrogen atmosphere, a mixture of methanol $(35 \mathrm{~mL})$, 1-bromo-3-(2hexenyl)benzene (9.24 g, $38.8 \mathrm{mmol})$ and $\mathrm{PtO}_{2}(158.5 \mathrm{mg}, 1.8 \mathrm{~mol} \%)$ was stirred for $5 \mathrm{~h}$ at room temperature. Then $\mathrm{PtO}_{2}$ was removed by filtration, and the solution concentrated. Flash chromatography over silica gel ( $n$-hexane) gave 1-bromo-3-hexylbenzene $(6.25 \mathrm{~g}, 95 \%) .{ }^{1} \mathrm{H}$ NMR (400 MHz, $\left.\mathrm{CDCl}_{3}\right) \delta 0.88(3 \mathrm{H}, \mathrm{t}, J=7.2 \mathrm{~Hz}), 1.25-1.35(6 \mathrm{H}, \mathrm{m}), 1.58$ (2H, quintet, $J=$ $7.2 \mathrm{~Hz}$ ), 2.56 (2H, t, $J=8.0 \mathrm{~Hz}), 7.08(1 \mathrm{H}, \mathrm{d}, J=7.2 \mathrm{~Hz}), 7.12(1 \mathrm{H}, \mathrm{t}, J=8.0 \mathrm{~Hz}), 7.29$ (1H, d, $J$ $=7.6 \mathrm{~Hz}), 7.32(1 \mathrm{H}, \mathrm{s}) .{ }^{13} \mathrm{C}-\mathrm{NMR}\left(100 \mathrm{MHz}, \mathrm{CDCl}_{3}\right) \delta 14.2,22.7,29.0,31.3,31.7,35.7,122.1$, 126.9, 128.5, 129.6, 131.2, 145.1. IR (neat) 2955, 2928, 2856, 1594, 1567, 1470, 1424, 1071, 776, $691 \mathrm{~cm}^{-1}$. MS (EI) m/z $242\left(\mathrm{M}^{+}+2,27\right), 240\left(\mathrm{M}^{+}, 27\right), 172\left(\mathrm{M}^{+}-68,68\right), 171\left(\mathrm{M}^{+}-69,41\right)$, $170\left(\mathrm{M}^{+}-70,69\right), 169\left(\mathrm{M}^{+}-71,37\right), 91\left(\mathrm{M}^{+}-149,100\right), 43\left(\mathrm{M}^{+}-197,54\right)$. HRMS. Calcd for $\mathrm{C}_{12} \mathrm{H}_{17} \mathrm{Br}: 240.0513$. Found: 240.0541. 
1-Bromo-2-hexylbenzene and 1-bromo-4-hexylbenzene were also synthesized by this method.

1-Bromo-2-hexylbenzene. ${ }^{1} \mathrm{H}-\mathrm{NMR}\left(400 \mathrm{MHz}, \mathrm{CDCl}_{3}\right) \delta 0.89(3 \mathrm{H}, \mathrm{t}, J=7.2 \mathrm{~Hz}), 1.29-1.41$ $(6 \mathrm{H}, \mathrm{m}), 1.60$ (2H, quintet, $J=7.6 \mathrm{~Hz}), 2.71(2 \mathrm{H}, \mathrm{t}, J=8.0 \mathrm{~Hz}), 7.00-7.04(1 \mathrm{H}, \mathrm{m}), 7.18-7.23$ (2H, m), $7.51(1 \mathrm{H}, \mathrm{d}, J=8.0 \mathrm{~Hz}) .{ }^{13} \mathrm{C}-\mathrm{NMR}\left(100 \mathrm{MHz}, \mathrm{CDCl}_{3}\right) \delta 14.2,22.7,29.1,30.0,31.7$, 36.3, 124.3, 127.1, 127.1, 130.1, 132.5, 141.9. IR (neat) 2955, 2927, 2857, 1566, 1469, 1438, 1377, 1024, 748, $658 \mathrm{~cm}^{-1}$. MS (EI) m/z $242\left(\mathrm{M}^{+}+2,22\right), 240\left(\mathrm{M}^{+}, 23\right), 172\left(\mathrm{M}^{+}-68,24\right), 171$ $\left(\mathrm{M}^{+}-69,38\right), 170\left(\mathrm{M}^{+}-70,25\right), 169\left(\mathrm{M}^{+}-71,36\right), 91\left(\mathrm{M}^{+}-149,100\right), 43\left(\mathrm{M}^{+}-197,57\right)$. HRMS. Calcd for $\mathrm{C}_{12} \mathrm{H}_{17} \mathrm{Br}: 240.0513$. Found: 240.0512 .

1-Bromo-4-hexylbenzene. ${ }^{9}{ }^{1} \mathrm{H}-\mathrm{NMR}\left(400 \mathrm{MHz}, \mathrm{CDCl}_{3}\right) \delta 0.87$ (3H, t, $\left.J=6.4 \mathrm{~Hz}\right), 1.24-1.32$ $(6 \mathrm{H}, \mathrm{m}), 1.57$ (2H, quintet, $J=7.2 \mathrm{~Hz}), 2.54(2 \mathrm{H}, \mathrm{t}, J=10.8 \mathrm{~Hz}), 7.40(2 \mathrm{H}, \mathrm{d}, J=8.0 \mathrm{~Hz}), 7.37$ $(2 \mathrm{H}, \mathrm{d}, J=8.0 \mathrm{~Hz}) .{ }^{13} \mathrm{C}$ NMR $\left(100 \mathrm{MHz} \mathrm{CDCl}_{3}\right) \delta 14.2,22.7,28.9,31.3,31.7,35.4,119.1$, 130.0, 131.0, 141.6. IR (neat) 2955, 2927, 2856, 1487, 1465, 1072, 1011, $801 \mathrm{~cm}^{-1}$. MS (EI) m/z 242 ( $\left.\mathrm{M}^{+}+2,46\right), 240\left(\mathrm{M}^{+}, 47\right), 171\left(\mathrm{M}^{+}-69,98\right), 169\left(\mathrm{M}^{+}-71,100\right), 91\left(\mathrm{M}^{+}-149,46\right), 43\left(\mathrm{M}^{+}-197\right.$, 27). HRMS. Calcd for $\mathrm{C}_{12} \mathrm{H}_{17} \mathrm{Br}$ : 240.0513. Found: 240.0525.

Preparation of 1-bromo-3-isobutylbenzene. Under an argon atmosphere, a $1.6 \mathrm{M}$ hexane solution of $n$-BuLi (14.5 mL, $23.3 \mathrm{mmol})$ was added to a THF solution (40 mL) of 2propyltriphenylphosphonium bromide $(5.70 \mathrm{~g}, 20 \mathrm{mmol})$ at $-78^{\circ} \mathrm{C}$. After stirring for $1 \mathrm{~h}$ at room temperature, $m$-bromobenzaldehyde $(1.94 \mathrm{~mL}, 16.6 \mathrm{mmol})$ was added at $-78{ }^{\circ} \mathrm{C}$, and the mixture was stirred for $1 \mathrm{~h}$ at room temperature. Then saturated $\mathrm{NH}_{4} \mathrm{Cl}$ aq. was added. The organic materials were extracted three times with $n$-hexane, dried over $\mathrm{MgSO}_{4}$ and concentrated. Flash chromatography over silica gel ( $n$-hexane) gave 1-bromo-3-(2-methyl-1-propenyl)benzene (1.80 g, 51\%). To a methanol solution $(5.0 \mathrm{~mL})$ of 1-bromo-3-(2-methyl-1-propenyl)benzene (210.0 mg, $1.0 \mathrm{mmol}$ ) was added $\mathrm{PtO}_{2}(92 \mathrm{mg}, 1.8 \mathrm{~mol} \%)$, and the mixture was stirred for $5 \mathrm{~h}$ under a hydrogen atmosphere at room temperature. $\mathrm{Pt} / \mathrm{PtO}_{2}$ were removed by filtration, and the solution concentrated. Flash chromatography over silica gel ( $n$-hexane) gave 1-bromo-3-isobutylbenzene (72.6 mg, 34\%). ${ }^{1} \mathrm{H}$ NMR (400 MHz, $\left.\mathrm{CDCl}_{3}\right) \delta 0.89(6 \mathrm{H}, \mathrm{d}, J=6.8 \mathrm{~Hz}), 1.84(1 \mathrm{H}$, septet, $J=6.8$ $\mathrm{Hz}), 2.43$ (2H, d, $J=6.4 \mathrm{~Hz}), 7.05(1 \mathrm{H}, \mathrm{d}, J=7.2 \mathrm{~Hz}), 7.13$ (1H, t, $J=8.0 \mathrm{~Hz}), 7.29(1 \mathrm{H}, \mathrm{s}), 7.30$ $(1 \mathrm{H}, \mathrm{d}, J=8.4 \mathrm{~Hz}) .{ }^{13} \mathrm{C}-\mathrm{NMR}\left(100 \mathrm{MHz}, \mathrm{CDCl}_{3}\right) \delta 22.4,30.2$, 45.0, 122.0, 127.6, 128.6, 129.4, 131.9, 143.8. IR (neat) 2955, 2924, 2868, 1591, 1566, 1471, 1425, 1073, 770, 693, $669 \mathrm{~cm}^{-1} . \mathrm{MS}$ (EI) $\mathrm{m} / \mathrm{z} 214\left(\mathrm{M}^{+}+2,50\right), 212\left(\mathrm{M}^{+}, 51\right), 172\left(\mathrm{M}^{+}-40,86\right), 171\left(\mathrm{M}^{+}-41,62\right), 170\left(\mathrm{M}^{+}-42,87\right), 169$ ( $\mathrm{M}^{+}-43$, 58), $91\left(\mathrm{M}^{+}-121,58\right), 43\left(\mathrm{M}^{+}-169,100\right)$. HRMS. Calcd for $\mathrm{C}_{10} \mathrm{H}_{13} \mathrm{Br}$ : 212.0200 Found: 212.0241.

1-Bromo-2-isobutylbenzene and 1-bromo-4-isobutylbenzene were also synthesized by this method.

1-Bromo-2-isobutylbenzene. ${ }^{1} \mathrm{H}$ NMR $\left(400 \mathrm{MHz}, \mathrm{CDCl}_{3}\right) \delta 0.93(6 \mathrm{H}, \mathrm{d}, J=6.4 \mathrm{~Hz}), 0.98(1 \mathrm{H}$, septet, $J=7.2 \mathrm{~Hz}), 2.60(2 \mathrm{H}, \mathrm{d}, J=7.2 \mathrm{~Hz}), 7.04(1 \mathrm{H}, \mathrm{t}, J=7.2 \mathrm{~Hz}), 7.16(1 \mathrm{H}, \mathrm{d}, J=7.2 \mathrm{~Hz})$, $7.21(1 \mathrm{H}, \mathrm{t}, J=7.2 \mathrm{~Hz}), 7.52(1 \mathrm{H}, \mathrm{d}, J=8.0 \mathrm{~Hz}) .{ }^{13} \mathrm{C}-\mathrm{NMR}\left(100 \mathrm{MHz}, \mathrm{CDCl}_{3}\right) \delta 22.4,28.8$, 45.2, 124.7, 126.8, 127.2, 131.1, 132.6, 140.8. IR (neat) 2956, 2927, 2867, 1566, 1468, 1438, 1383, 1366, 1166, 1076, 1021, 746, $659 \mathrm{~cm}^{-1}$. MS (EI) m/z $214\left(\mathrm{M}^{+}+2,46\right), 212\left(\mathrm{M}^{+}, 47\right), 172$ $\left(\mathrm{M}^{+}-40,90\right), 171\left(\mathrm{M}^{+}-41,100\right), 170\left(\mathrm{M}^{+}-42,91\right), 169\left(\mathrm{M}^{+}-43,97\right), 133\left(\mathrm{M}^{+}-79,32\right), 91\left(\mathrm{M}^{+}-121\right.$, 
95), 90 ( $\left.\mathrm{M}^{+}-122,48\right), 89\left(\mathrm{M}^{+}-123,38\right), 43\left(\mathrm{M}^{+}-169\right.$, 85). HRMS. Calcd for $\mathrm{C}_{10} \mathrm{H}_{13} \mathrm{Br}: 212.0200$ Found: 212.0211.

1-Bromo-4-isobutylbenzene. ${ }^{1} \mathrm{H}$ NMR $\left(400 \mathrm{MHz}, \mathrm{CDCl}_{3}\right) \delta 0.88(6 \mathrm{H}, \mathrm{d}, J=6.8 \mathrm{~Hz}), 1.82(1 \mathrm{H}$, septet, $J=6.8 \mathrm{~Hz}), 2.42(2 \mathrm{H}, \mathrm{d}, J=7.2 \mathrm{~Hz}), 7.01(2 \mathrm{H}, \mathrm{d}, J=8.0 \mathrm{~Hz}), 7.37(2 \mathrm{H}, \mathrm{d}, J=8.0 \mathrm{~Hz})$. ${ }^{13} \mathrm{C} \mathrm{NMR}\left(100 \mathrm{MHz}, \mathrm{CDCl}_{3}\right) \delta 22.3,30.2,44.8,119.2,130.6,130.9,140.4$. IR (neat) 2955, 2923, 2868, 1591, 1488, 1466, 1073, 1012, 839, $785 \mathrm{~cm}^{-1}$. MS (EI) m/z $214\left(\mathrm{M}^{+}+2,32\right), 212$ $\left(\mathrm{M}^{+}\right.$, 32), $172\left(\mathrm{M}^{+}-40,34\right), 171\left(\mathrm{M}^{+}-41,98\right), 170\left(\mathrm{M}^{+}-42,35\right), 169\left(\mathrm{M}^{+}-43,100\right), 91\left(\mathrm{M}^{+}-121\right.$, 27), 90 ( $\left.\mathrm{M}^{+}-122,28\right), 43$ ( $\mathrm{M}^{+}-169$, 38). HRMS. Calcd for $\mathrm{C}_{10} \mathrm{H}_{13} \mathrm{Br}$ : 212.0200 Found: 212.0200.

Bromotoluene (mixture of isomers). The structures of the products were determined by comparison with authentic commercial samples.

Bromoethylbenzenes (mixture of isomers). The structures of 1-bromo-2-ethylbenzene and 1bromo-4-ethylbenzene were determined by comparison with authentic commercial samples. For 1-bromo-3-ethylbenzene, see ref. 10.

Bromooctylbenzenes (mixture of isomers). The structures were determined by analogy with bromohexylbenzenes. For 1-bromo-4-octylbenzene, see ref. 11. ${ }^{1} \mathrm{H}$ NMR (400 $\left.\mathrm{MHz}, \mathrm{CDCl}_{3}\right) \delta$ $0.88(9 \mathrm{H}, \mathrm{t}, J=7.2 \mathrm{~Hz}, o, m, p)$, 1.26-1.29 (30H, m, o, $m, p)$, 1.53-1.62 (6H, m, o, $m, p$ ), 2.52$2.58(4 \mathrm{H}, \mathrm{m}, m, p), 2.71(2 \mathrm{H}, \mathrm{t}, J=8.0 \mathrm{~Hz}, o), 7.00-7.05(\mathrm{~m}, 1 \mathrm{H}, o, 2 \mathrm{H}, p), 7.08(1 \mathrm{H}, \mathrm{d}, J=7.4$ $\mathrm{Hz}, m), 7.13(1 \mathrm{H}, \mathrm{t}, J=7.2 \mathrm{~Hz}, m), 7.19-7.21(2 \mathrm{H}, \mathrm{m}, o), 7.30(1 \mathrm{H}, \mathrm{d}, J=8.0 \mathrm{~Hz}, m), 7.32(1 \mathrm{H}$, s, m), $7.37(2 \mathrm{H}, \mathrm{d}, J=8.0 \mathrm{~Hz}, p), 7.51(1 \mathrm{H}, \mathrm{d}, J=8.0 \mathrm{~Hz}, o) \cdot{ }^{13} \mathrm{C}$ NMR $\left(100 \mathrm{MHz}, \mathrm{CDCl}_{3}\right) \delta$ 14.3 (o, $m, p), 22.8$ ( $m, p), 22.8$ (o), 29.3 (o, $m, p), 29.3$ (o, $m, p), 29.4$ ( $m, p), 29.5$ (o), 30.1 $(o), 31.4(m), 31.4(p), 32.0(m, p), 32.0(o), 35.5(p), 35.7(m), 36.3(o), 119.1(p), 122.2$ $(\mathrm{m}), 124.3(\mathrm{o}), 126.9(\mathrm{~m}), 127.1(\mathrm{o}), 127.2$ (o), 128.5 (m), 129.6 (m), 130.0 (o), 130.1 (o), $131.1(p), 131.3(m), 132.6(o), 141.7(p), 142.0(o), 145.1(m)$. IR (neat) 2954, 2925, 2855, 1595, 1568, 1488, 1469, 1072, 1023, 1011, 777, 748, $692 \mathrm{~cm}^{-1}$. MS (EI) m/z $270\left(\mathrm{M}^{+}+2,17\right), 268$ $\left(\mathrm{M}^{+}, 17\right), 172\left(\mathrm{M}^{+}-96,28\right), 171\left(\mathrm{M}^{+}-97,37\right), 170\left(\mathrm{M}^{+}-98,29\right), 169\left(\mathrm{M}^{+}-99,37\right), 91\left(\mathrm{M}^{+}-177\right.$, 100), $57\left(\mathrm{M}^{+}-211,78\right), 43\left(\mathrm{M}^{+}-225,60\right)$. HRMS. Calcd for $\mathrm{C}_{14} \mathrm{H}_{21} \mathrm{Br}$ : 268.0826. Found: 268.0799. Anal. Calcd for $\mathrm{C}_{14} \mathrm{H}_{21} \mathrm{Br}$ : C; 62.46, H; 7.86, Br; 29.68\%. Found: C; 61.98, H; 8.03, Br; 29.76\%.

Bromoisobutylbenzenes (mixture of isomers). ${ }^{1} \mathrm{H}$ NMR $\left(400 \mathrm{MHz}, \mathrm{CDCl}_{3}\right) \delta 0.89(6 \mathrm{H}, \mathrm{d}, J=$ $6.8 \mathrm{~Hz}, p), 0.89(6 \mathrm{H}, \mathrm{d}, J=6.8 \mathrm{~Hz}, m), 0.93(6 \mathrm{H}, \mathrm{d}, J=6.4 \mathrm{~Hz}, o), 1.79-1.90$ (2H, m, $m, p), 1.99$ $(1 \mathrm{H}$, septet, $J=7.2 \mathrm{~Hz}, o), 2.41-2.44(4 \mathrm{H}, \mathrm{m}, m, p), 2.60(2 \mathrm{H}, \mathrm{d}, J=7.2 \mathrm{~Hz}, o), 7.00(2 \mathrm{H}, \mathrm{d}, J=$ $8.0 \mathrm{~Hz}, p), 7.01-7.06(2 \mathrm{H}, \mathrm{m}, o, m), 7.13(1 \mathrm{H}, \mathrm{t}, J=8.0 \mathrm{~Hz}, m), 7.16(1 \mathrm{H}, \mathrm{d}, J=8.0 \mathrm{~Hz}, o), 7.21$ $(1 \mathrm{H}, \mathrm{t}, J=7.6 \mathrm{~Hz}, o), 7.29(1 \mathrm{H}, \mathrm{s}, m), 7.30(1 \mathrm{H}, \mathrm{d}, J=8.4 \mathrm{~Hz}, m), 7.37(2 \mathrm{H}, \mathrm{d}, J=8.0 \mathrm{~Hz}, p)$, $7.52(1 \mathrm{H}, \mathrm{d}, J=8.4 \mathrm{~Hz}, o) .{ }^{13} \mathrm{C}-\mathrm{NMR}\left(100 \mathrm{MHz}, \mathrm{CDCl}_{3}\right) \delta 22.4(o), 22.4(\mathrm{~m}), 22.4(p), 28.9$ (o), 30.2 (m,p), $44.8(p), 45.1(m), 45.2(o), 119.2(p), 122.1(m), 124.7(o), 126.8(o), 127.2$ $(o), 127.6(m), 128.6(m), 129.5(m), 130.7(p), 131.0(p), 131.2(o), 131.9(m), 132.6(o)$, $140.4(p), 140.8(o), 143.9$ (m). IR (neat) 2955, 2925, 2868, 1592, 1567, 1488, 1469, 1384, 1366, 1167, 1074, 1021, 841, 771, 747, 693, $669 \mathrm{~cm}^{-1}$. MS (EI) m/z $214\left(\mathrm{M}^{+}+2,62\right), 212\left(\mathrm{M}^{+}\right.$, 63), 172 ( $\left.\mathrm{M}^{+}-40,90\right), 171\left(\mathrm{M}^{+}-41,100\right), 170$ ( $\left.\mathrm{M}^{+}-42,93\right), 169\left(\mathrm{M}^{+}-43,95\right), 91\left(\mathrm{M}^{+}-121,48\right), 43$ 
( $\mathrm{M}^{+}-169$, 90). HRMS. Calcd for $\mathrm{C}_{10} \mathrm{H}_{13} \mathrm{Br}$ : 212.0200. Found: 212.0187. Anal. Calcd for $\mathrm{C}_{10} \mathrm{H}_{13} \mathrm{Br}$ : C; 56.36, H; 6.15, Br; 37.49\%. Found: C; 56.12, H; 6.08, Br; 37.52\%.

Bromoneopentylbenzenes (mixture of isomers). The structures of all the isomers were determined by analogy with bromo-isobutylbenzenes. For 1-bromo-2-neopentylbenzene and 1bromo-4-neopentyl-benzene, see ref. 12. ${ }^{1} \mathrm{H}-\mathrm{NMR}\left(400 \mathrm{MHz}, \mathrm{CDCl}_{3}\right) \delta 0.89(9 \mathrm{H}, \mathrm{s}, p), 0.90$ (9H, s, m), $0.97(9 \mathrm{H}, \mathrm{s}, o), 2.44(2 \mathrm{H}, \mathrm{s}, p), 2.45(2 \mathrm{H}, \mathrm{s}, m), 2.74(2 \mathrm{H}, \mathrm{s}, o), 6.99(2 \mathrm{H}, \mathrm{d}, J=8.8$ $\mathrm{Hz}, p), 7.00-7.03(1 \mathrm{H}, \mathrm{m}, o) 7.04(1 \mathrm{H}, \mathrm{d}, J=7.6 \mathrm{~Hz}, m), 7.13(1 \mathrm{H}, \mathrm{t}, J=7.2 \mathrm{~Hz}, m), 7.20-7.21$ (2H, m, o), $7.27(1 \mathrm{H}, \mathrm{s}, m), 7.33(1 \mathrm{H}, \mathrm{d}, J=8.0 \mathrm{~Hz}, m), 7.38(2 \mathrm{H}, \mathrm{d}, J=8.0 \mathrm{~Hz}, p), 7.54(1 \mathrm{H}, \mathrm{d}$, $J=8.0 \mathrm{~Hz}, o) .{ }^{13} \mathrm{C}-\mathrm{NMR}\left(100 \mathrm{MHz}, \mathrm{CDCl}_{3}\right) \delta 29.4,29.4,29.7,31.8,31.9,33.3,48.0,49.6,49.9$, 119.6, 121.6, 125.9, 126.4, 127.3, 128.7, 128.9, 129.0, 130.5, 132.0, 132.2, 132.8, 133.1, 138.5, 139.2, $141.9 \mathrm{~cm}^{-1}$. IR (neat) 2953, 2865, 1592, 1567, 1488, 1474, 1423, 1393, 1364, 1236, 1205 , 1073, 997, 890, 841, 804, 785, 741, 696. MS (EI) m/z $228\left(\mathrm{M}^{+}+2,10\right), 226\left(\mathrm{M}^{+}, 11\right), 172\left(\mathrm{M}^{+}-\right.$ 54, 19), $170\left(\mathrm{M}^{+}-56,20\right), 57\left(\mathrm{M}^{+}-169,100\right)$. HRMS. Calcd for $\mathrm{C}_{11} \mathrm{H}_{15} \mathrm{Br}$ : 226.0357. Found: 226.0346. Anal. Calcd for $\mathrm{C}_{11} \mathrm{H}_{15} \mathrm{Br}$ : C; 58.17, H; 6.66, Br; 35.18\%. Found: C; 57.93, H; 6.71, Br; 35.01\%.

2-Bromo-1,3-dimethylbenzene, 1-bromo-2,4-dimethylbenzene and 1-bromo-3,5 dimethylbenzene. Structures determined by comparison with commercial authentic samples.

1-Bromo-2,5-dimethylbenzene and 1,4-dibromo-2,5-dimethylbenzene. Structures determined by comparison with commercial authentic samples.

Dibromo-1,3-dimethylbenzene (mixture of isomers). For 2,5-dibromo-1,3-dimethylbenzene and 4,6-dibromo-1,3-dimethylbenzene, see ref. 13. ${ }^{1} \mathrm{H}$ NMR (400 $\left.\mathrm{MHz}, \mathrm{CDCl}_{3}\right) \delta 2.30(6 \mathrm{H}, \mathrm{s}$, 4,6), 2.37 (3H, s, 2,4), 2.38 (6H, s, 2,5), 2.60 (6H, s, 2,4), $6.93(1 \mathrm{H}, \mathrm{d}, J=8.0 \mathrm{~Hz}, 2,4), 7.09(1 \mathrm{H}$, s, 4,6), $7.21(1 \mathrm{H}, \mathrm{s}, 2,5), 7.38(1 \mathrm{H}, \mathrm{d}, J=8.0 \mathrm{~Hz}, 2,4), 7.67(1 \mathrm{H}, \mathrm{s}, 4,6) .{ }^{13} \mathrm{C}-\mathrm{NMR}(100 \mathrm{MHz}$, $\left.\mathrm{CDCl}_{3}\right) \delta 22.3,23.7,24.2,24.4,120.1,121.8,126.1,127.9,128.7,130.6,130.7,132.4,134.7$, 136.6, 137.1, 137.6, 140.0. FT-IR (neat) 2978, 2952, 2920, 1560, 1456, 1378, 1261, 1122, 1051, 1029, 997, 854. MS (EI) m/z $266\left(\mathrm{M}^{+}+4,51\right), 264\left(\mathrm{M}^{+}+2,100\right), 262\left(\mathrm{M}^{+}, 55\right), 185\left(\mathrm{M}^{+}-77,68\right)$, $183\left(\mathrm{M}^{+}-79,72\right), 104\left(\mathrm{M}^{+}-158,53\right), 103\left(\mathrm{M}^{+}-159,59\right), 51\left(\mathrm{M}^{+}-211,48\right)$. HRMS. Calcd for $\mathrm{C}_{8} \mathrm{H}_{8} \mathrm{Br}_{2}$ : 261.8992. Found: 261.8980 .

1-Bromo-2,4,6-trimethylbenzene and 1,3-dibromo-2,4,6-trimethylbenzene. The structures of the products were determined by comparison with commercial authentic samples.

1,3,5-Tribromo-2,4,6-trimethylbenzene. ${ }^{14}{ }^{1} \mathrm{H}$ NMR (400 MHz, $\left.\mathrm{CDCl}_{3}\right) \delta 2.65$ (9H, s). ${ }^{13} \mathrm{C}$ NMR (100 MHz, $\left.\mathrm{CDCl}_{3}\right) \delta 26.4,124.8,136.8 . \mathrm{IR}(\mathrm{KBr}) 2919,1538,1434,1375,1349,1017$, 954, $647 \mathrm{~cm}^{-1}$. MS (EI) m/z $358\left(\mathrm{M}^{+}+4,97\right), 356\left(\mathrm{M}^{+}+2,100\right), 354\left(\mathrm{M}^{+}, 279\left(\mathrm{M}^{+}-75,50\right), 277\right.$ $\left(\mathrm{M}^{+}-77,95\right), 275\left(\mathrm{M}^{+}-79,50\right), 117\left(\mathrm{M}^{+}-237,56\right), 116\left(\mathrm{M}^{+}-238,66\right), 115\left(\mathrm{M}^{+}-239,72\right)$. HRMS. Calcd for $\mathrm{C}_{9} \mathrm{H}_{9} \mathrm{Br}_{3}$ : 353.8255. Found: 353.8283 .

1-Bromo-2,3,4-trimethylbenzene. ${ }^{14,15}{ }^{1} \mathrm{H}$ NMR (400 MHz, $\left.\mathrm{CDCl}_{3}\right) \delta 2.23$ (6H, s), 2.39 (3H, s), $6.84(1 \mathrm{H}, \mathrm{d}, J=8.0 \mathrm{~Hz}), 7.28(1 \mathrm{H}, \mathrm{d}, J=8.0 \mathrm{~Hz}) .{ }^{13} \mathrm{C} \mathrm{NMR}\left(100 \mathrm{MHz}, \mathrm{CDCl}_{3}\right) \delta 16.9,20.1$, 20.7, 122.7, 128.4, 129.2, 135.4, 135.4, 136.8. IR (neat) 2942, 1582, 1455, 1405, 1378, 1250, 1181, 1134, 1077, 1000, 893, 851, 826, $801 \mathrm{~cm}^{-1}$. MS (EI) m/z $200\left(\mathrm{M}^{+}+2,69\right), 198\left(\mathrm{M}^{+}, 72\right)$, 119 ( $\left.\mathrm{M}^{+}-79,100\right)$. HRMS. Calcd for $\mathrm{C}_{9} \mathrm{H}_{11} \mathrm{Br}: 198.0044$ Found: 198.0028. 
5-Bromo-1,2,3-trimethylbenzene. ${ }^{16}{ }^{1} \mathrm{H}-\mathrm{NMR}\left(400 \mathrm{MHz}, \mathrm{CDCl}_{3}\right) \delta 2.10(3 \mathrm{H}, \mathrm{s}), 2.24(6 \mathrm{H}, \mathrm{s})$, 7.14 (2H, s). ${ }^{13} \mathrm{C}$ NMR $\left(100 \mathrm{MHz}, \mathrm{CDCl}_{3}\right) \delta 15.1,20.5,20.7,118.1,129.9,133.8,138.3$. IR (neat) 2920, 1579, 1470, 1455, 1377, 1185, 1000, 851, $801 \mathrm{~cm}^{-1}$. MS (EI) m/z $200\left(\mathrm{M}^{+}+2\right.$, 59), $198\left(\mathrm{M}^{+}, 60\right), 119$ ( $\left.\mathrm{M}^{+}-79,100\right)$. HRMS. Calcd for $\mathrm{C}_{9} \mathrm{H}_{11} \mathrm{Br}$ : 198.0044 Found: 198.0046.

1,5-Dibromo-2,3,4-trimethylbenzene. ${ }^{17}{ }^{1} \mathrm{H}-\mathrm{NMR}\left(400 \mathrm{MHz}, \mathrm{CDCl}_{3}\right) \delta 2.30(3 \mathrm{H}, \mathrm{s}), 2.35(6 \mathrm{H}$, s), $7.64(1 \mathrm{H}, \mathrm{s}) .{ }^{13} \mathrm{C}-\mathrm{NMR}\left(100 \mathrm{MHz}, \mathrm{CDCl}_{3}\right) \delta 18.4,20.2,122.5,132.6,134.8,138.1 . \mathrm{IR}(\mathrm{KBr})$ 2920, 1557, 1440, 1415, 1378, 1160, 1008, 904, 856, $655 \mathrm{~cm}^{-1}$. MS (EI) m/z $280\left(\mathrm{M}^{+}+4,50\right)$, $278\left(\mathrm{M}^{+}+2,100\right), 276\left(\mathrm{M}^{+}, 53\right), 199\left(\mathrm{M}^{+}-77,57\right), 197\left(\mathrm{M}^{+}-79\right.$, 58). HRMS. Calcd for $\mathrm{C}_{9} \mathrm{H}_{10} \mathrm{Br}_{2}$ : 275.9149. Found: 375.9164.

1,2,3-Tribromo-4,5,6-trimethylbenzene. ${ }^{14}{ }^{1} \mathrm{H}-\mathrm{NMR}$ (400 MHz, $\mathrm{CDCl}_{3}$ ) $\delta 2.30$ (3H, s), 2.49 $(9 \mathrm{H}, \mathrm{s}) .{ }^{13} \mathrm{C}-\mathrm{NMR}\left(100 \mathrm{MHz}, \mathrm{CDCl}_{3}\right) \delta$ 19.0, 23.0, 125.5, 125.7, 136.2, 137.2. IR (KBr) 2919, 1430, 1371, 1354, 1232, 1004, 930, $657 \mathrm{~cm}^{-1}$. MS (EI) m/z $358\left(\mathrm{M}^{+}+4,96\right), 356\left(\mathrm{M}^{+}+2,100\right)$, $354\left(\mathrm{M}^{+}\right.$, 36), $277\left(\mathrm{M}^{+}-77,62\right), 115\left(\mathrm{M}^{+}-239,40\right)$. HRMS. Calcd for $\mathrm{C}_{9} \mathrm{H}_{9} \mathrm{Br}_{3}$ : 353.8254. Found: 353.8244.

3-Bromo-1,2,4,5-tetramethylbenzene. The structure of the product was determined by comparison with commercial authentic sample.

1,4-Dibromo-2,3,5,6-tetramethylbenzene. ${ }^{14}{ }^{1} \mathrm{H}-\mathrm{NMR}\left(400 \mathrm{MHz}, \mathrm{CDCl}_{3}\right) \delta 2.48(12 \mathrm{H}, \mathrm{s}) .{ }^{13} \mathrm{C}$ NMR (100 MHz, $\left.\mathrm{CDCl}_{3}\right) \delta 22.4,127.9,134.8 . \mathrm{IR}(\mathrm{KBr})$ 2922, 1414, 1381, 1173, 987, $690 \mathrm{~cm}^{-1}$. MS (EI) m/z 294 ( $\left.\mathrm{M}^{+}+4,50\right), 292$ ( $\left.\mathrm{M}^{+}+2,100\right), 290$ (M+ 53), 213 ( $\left.\mathrm{M}^{+}-77,69\right), 211\left(\mathrm{M}^{+}-79,74\right)$. HRMS. Calcd for $\mathrm{C}_{10} \mathrm{H}_{12} \mathrm{Br}_{2}$ : 289.9305. Found: 289.9333.

1-Bromo-2,3,4,5-tetramethylbenzene. ${ }^{14,15}{ }^{1} \mathrm{H}-\mathrm{NMR}\left(400 \mathrm{MHz}, \mathrm{CDCl}_{3}\right) \delta 2.14$ (3H, s), 2.22 (3H, s), 2.24 (3H, s), 2.37 (3H, s), 7.23 (1H, s). ${ }^{13} \mathrm{C}-\mathrm{NMR}\left(100 \mathrm{MHz}, \mathrm{CDCl}_{3}\right.$ ) $\delta$ 16.1, 17.4, 20.1, 20.5, 122.0, 130.6, 132.8, 134.0, 135.2, 136.6. IR (neat) 2922, 1460, 1379, 1199, 941, 859, 752 $\mathrm{cm}^{-1}$. MS (EI) m/z $214\left(\mathrm{M}^{+}+2,60\right), 212\left(\mathrm{M}^{+}, 62\right), 133\left(\mathrm{M}^{+}-79,100\right)$. HRMS. Calcd for $\mathrm{C}_{10} \mathrm{H}_{13} \mathrm{Br}$ : 212.0200. Found: 212.0191.

1,2-Dibromo-3,4,5,6-tetramethylbenzene. ${ }^{14}{ }^{1} \mathrm{H}-\mathrm{NMR}$ (400 MHz, $\mathrm{CDCl}_{3}$ ) $\delta 2.24$ (6H, s), 2.49 $(6 \mathrm{H}, \mathrm{s}) .{ }^{13} \mathrm{C}-\mathrm{NMR}\left(100 \mathrm{MHz}, \mathrm{CDCl}_{3}\right) \delta 17.9,22.8,125.3,135.2,135.4 . \mathrm{IR}(\mathrm{KBr}) 2918,1374$, 1255, 1193, 992, 951, 889, $770 \mathrm{~cm}^{-1}$. MS (EI) m/z $294\left(\mathrm{M}^{+}+4,51\right), 292\left(\mathrm{M}^{+}+2,100\right), 290\left(\mathrm{M}^{+}\right.$, 53), $213\left(\mathrm{M}^{+}-77,55\right), 211\left(\mathrm{M}^{+}-79\right.$, 56). HRMS. Calcd for $\mathrm{C}_{10} \mathrm{H}_{12} \mathrm{Br}_{2}$ : 289.9305. Found: 289.9316.

\section{Acknowledgments}

M. A. expresses her thanks to JSPS for a Grant-in-Aid for Encouragement of Young Scientists (No. 13771323), and the Hayashi Memorial Foundation for Female Natural Scientists.

\section{References}


1. Taylor, R. Electrophilic Aromatic Substitution; John Wiley \& Sons: New York, 1990.

2. For example, (a) Acrec, S. F. Chem. Ber. 1904, 37, 994.(b) Bigelow, L. A.; Jonson, J. R.; Sandboan, L. T. Org. Syn. 1941 Coll. Vol. I, 133. (c) Compernolle, F.; Toppet, S. J. Heterocycl. Chem. 1986, 23, 541.

3. (a) Olah, G. A.; Tolgyesi, W. S.; Dear, R. E. A. J. Org. Chem. 1962, 27, 3464. (b) Crump, J. W.; Gornowicz. G. A. J. Org. Chem. 1963, 28, 949. (c) De Valois, P. J.; Van Albada, M. P.; Veenland, J. U. Tetrahedron 1968, 24, 1835. (d) Olah, G. A.; Lapierre, J. C; Carlson, C. G. J. Org. Chem. 1965, 30, 541. (e) Olah, G. A.; Tolgyesi, W. S.; Dear, R. E. A. J. Org. Chem. 1962, 27, 3441. (f) Olah, G. A.; Tolgyesi, W. S.; Dear, R. E. A. J. Org. Chem. 1962, 27, 3449. (g) Olah, G. A.; Tolgyesi, W. S.; Dear, R. E. A. J. Org. Chem. 1962, 27, 3455.

4. One-pot $m$-bromination reaction of toluene using a stoichiometric amount of $\mathrm{AlBr}_{3}$ has been reported. Brown, H. C.; McGary, C. W. J. Am. Chem. Soc. 1955, 77, 2306.

5. (a) Yonehara, F.; Kido, Y.; Morita, S.; Yamaguchi, M. J. Am. Chem. Soc. 2001, 123, 11310.

(b) Yonehara, F.; Kido, Y.; Yamaguchi, M. Chem. Commun. 2000, 1189.

6. (a) Kido, Y.; Yonehara, F.; Yamaguchi, M. Tetrahedron 2001, 57, 827. (b) Kido, Y.; Arisawa, M.; Yamaguchi, M. J. Synth. Org. Chem. Jpn. 2000, 58, 1030. (c) Kido, Y.; Yoshimura, S.; Yamaguchi, M.; Uchimaru, T. Bull. Chem. Soc. Jpn. 1999, 72, 1445. (d) Kido, Y.; Yamaguchi, M. J. Org. Chem. 1998, 63, 8086. (e) Yamaguchi, M.; Kido, Y.; Hayashi, A.; Hirama, M. Angew., Chem. Int. Ed. Engl. 1997, 36, 1313.

7. (a) Kobayashi, K.; Arisawa, M.; Yamaguchi, M. J. Am. Chem. Soc. 2002, 124, 8528. (b) Arisawa, M.; Amemiya, R.; Yamaguchi, M. Org. Lett. 2002, 4, 2209. (c) Arisawa, M.; Miyagawa, C.; Yamaguchi, M. Synthesis 2002, 138. (d) Arisawa, M.; Miyagawa, C.; Yoshimura, S.; Kido, Y.; Yamaguchi, M. Chem. Lett. 2001, 1080. (e) Arisawa, M.; Akamatsu, K.; Yamaguchi, M. Org. Lett. 2001, 3, 789. (f) Yamaguchi, M.; Tsukagoshi, T.; Arisawa, M. J. Am. Chem. Soc. 1999, 121, 4074. (g) Kobayashi, K.; Arisawa, M.; Yamaguchi, M. Inorg. Chim. Acta 1999, 296, 67. (h) Yamaguchi, M.; Sotokawa, T.; Hirama, M. Chem. Commun. 1997, 743.

8. Gruber, R.; Kirsch, G.; Cagniant, D. Bull. Soc. Chim. Fr. 1987, 498.

9. Franks, S; Hartley, F. R. J. Chem. Soc., Perkin Trans. 1 1980, 2233.

10. Lunazzi, L.; Mazzanti, A.; Alverez, M. J. Org. Chem. 2000, 65, 3200.

11. Peng, Z.; Gharavi, A. R.; Yu, L. J. Am. Chem. Soc. 1997, 119, 4622.

12. Hellwinkel, D.; Knabe, B. Chem. Ber. 1971, 104, 1761. Archer, W. J.; Hossaini, M. A.; Taylor, R. J. Chem. Soc., Perkin Trans. 2 1982, 181.

13. Itoh, T.; Matsuda, K.; Iwamura, H.; Hori, K. J. Am. Chem. Soc. 2000, 122, 2567. Allinson, G.; Bushby, R. J.; Jesudason, M. V.; Paillaud, J.; Taylor N. J. Chem. Soc., Perkin Trans. 2 1997, 147.

14. Kajigaeshi, S.; Kakinami, T.; Moriwaki, M.; Tanaka, T.; Fujisaki, S.; Okamoto, T. Bull. Chem. Soc. Jpn. 1989, 62, 439.

15. Mitchell, R. H.; Lai, Y.; Williams, R. V. J. Org. Chem. 1979, 44, 4733. 
16. Baciocchi, E.; Dalla, C. A.; Eberson, L.; Mandolini, L.; Roi, C. J. Org. Chem. 1986, 51, 4544.

17. Suzuki, H.; Mishina, T.; Hanafusa, T. Bull. Chem. Soc. Jpn. 1978, 51, 3099. 